\title{
Is there an increased risk of an infected prosthetic joint after cystoscopy or transurethral prostatectomy?
}

\author{
Nahid Punjani ${ }^{1}$; J. Andrew McClure ${ }^{2}$; Brent Lanting ${ }^{3}$; Blayne Welk ${ }^{1,2,4}$ \\ ${ }^{1}$ Division of Urology, Department of Surgery, Western University, London, ON, Canada; ${ }^{2}$ Institute for \\ Clinical Evaluative Sciences, ON, Canada; ${ }^{3}$ Division of Orthopedics, Department of Surgery, Western \\ University, London, ON, Canada; ${ }^{4}$ Department of Epidemiology and Biostatistics, Western University, \\ London, ON, Canada
}

Acknowledgements: This project was conducted at the Institute for Clinical Evaluative Sciences (ICES) Western Site. The ICES is funded by an annual grant from the Ontario Ministry of Health and Long-term Care. ICES Western is funded by an operating grant from the Academic Medical Organization of Southwestern Ontario. Disclaimer: The opinions, results, and conclusions are those of the authors, and no endorsement by the Institute for Clinical Evaluative Sciences, Ontario Ministry of Health and Long-Term Care, or Academic Medical Organization of Southwestern Ontario is intended or should be inferred. Parts of this material are based on data and information compiled and provided by CIHI. However, the analyses, conclusions, opinions and statements expressed herein are those of the author, and not necessarily those of CIHI.

Cite as: Can Urol Assoc J 2018 November 5; Epub ahead of print. http://dx.doi.org/10.5489/cuaj.5721

Published online November 5, 2018

$* * *$

\section{Introduction}

Prosthetic joint infections are associated with significant cost, morbidity, and potential mortality. (1) Urological antibiotic prophylaxis guidelines for individuals with a prosthetic knee or hip joint suggests that prophylaxis is necessary in patients with higherrisk genitourinary procedures that are performed within the first two years after prosthetic joint placement in order to minimize the risk of joint infection. (2) Both cystoscopy (a “low-risk" procedure) and transurethral prostatectomy (TURP, a "high-risk” procedure) can cause symptomatic infections; however, there is an obvious contrast in the invasiveness of these two procedures (especially with contemporary flexible cystoscopes). $(3,4)$ Given that there is only limited clinical evidence to support antibiotic prophylaxis after joint replacement in general, and the fact that many patients receive prophylactic antibiotics with low-risk procedures out of an abundance of caution,(5) we sought to determine the risk of prosthetic joint infection that is associated with both cystoscopy and TURP. 


\section{Methods}

We conducted a retrospective, population-based, cohort study using administrative databases from the province of Ontario, Canada. We identified all individuals who were $>66$ years of age and underwent a total hip arthroplasty (THA) or total knee arthroplasty (THA) between April 1, 2003 and December 31, 2013. A full description of our data sources, methodology, covariates and coding definitions are included in our prior work. (6) Similar to our prior study, the primary outcome was hospital admission for a THA/TKA joint infection that occurred within 2 years of the initial joint replacement. Using the Ontario Health Insurance Plan and hospital discharge records we identified our two exposures of interest: cystoscopy (measured as a cumulative count variable to account for multiple procedures) or TURP (only the first one was considered, and joint infection was required to occur within 90 days to maintain biologic plausibility; men with a prior TURP where excluded). For our primary analysis we performed multivariate Cox proportional hazards with subdistribution modelling (including covariates with potentially clinically relevant differences between the exposed and unexposed groups). We accounted for the time-varying nature of the exposures, and treated additional joint replacements or death as competing events. Patients were censored at the time of death, additional joint replacement, emigration from the province, or at the end of the at-risk exposure window or study period (2.25 years from joint replacement). A secondary analysis examined the risk among patients who filled a prescription for an antibiotic $+/-3$ days of their cystoscopy. Results are reported as hazard ratios (HR) with 95\% confidence intervals and p-values. A two sided $\mathrm{p}<0.05$ was considered significant.

\section{Results}

We identified 113,061 people who underwent a prosthetic joint replacement (THA, $\mathrm{n}=44,495$ and TKA, $n=68,566$, Table 1 ). When comparing those who underwent a cystoscopy ( $n=8,426,7.5 \%)$ to those who did not (104,635, 92.5\%), patients who had cystoscopy were slightly older, had less comorbidities, and were more likely to have a history of previous cystoscopies, UTI, and prior antibiotic exposure; these patients were also more likely to have previous Urology clinic encounters, hospitalizations and ER visits. They underwent a median of 1 (interquartile range 1-2) cystoscopies during the observation period. In our primary adjusted analysis, the risk of joint infection was not significantly associated with cystoscopy (HR 1.05, 95\% CI 0.85-1.30, p=0.66; Table 2). In our secondary analysis, 2,712 (32\%) patients filled a prescription for an antibiotic at the time of cystoscopy. There was no association between cystoscopy and joint infection, regardless of antibiotic exposure.

There were 43,461 male patients who underwent a THA/TKA, of which 1,095 (2.5\%) underwent a TURP. Men who underwent a TURP were more likely to have had previous urinary retention/cystoscopy/urinary infection, and a higher number of Urology 
and ER visits. In multivariable analysis, TURP was associated with a significant increase in the risk of joint infection (HR 3.42, 95\%CI 1.29-9.10, $\mathrm{p}=0.01$; Table 2).

\section{Discussion}

We demonstrated that cystoscopy is not significantly related to periprosthetic joint infection, even when accounting for patients who were not prescribed outpatient oral antibiotics at the time of cystoscopy. In contrast, a TURP was associated with a significantly increased risk of periprosthetic joint infection within 90 days, however it is important to note that the absolute risk remains low (0.5\%). It is likely that this risk is present despite periprocedural antibiotics, as these would be expected in the majority of patients undergoing a TURP. $(5,7)$ The Canadian Urologic Association guidelines on antibiotic prophylaxis do not directly address prosthetic joint patients(7), and the previous statement by the American Urology Association regarding antibiotic prophylaxis is now 15 years old.(2) We hope this study will help urologists and orthopedic surgeons practice good antimicrobial stewardship, assist with patient counselling in the post-joint replacement period, and contribute to future guidelines on antibiotic prophylaxis in urology.

Limitations of our research include the inability to accurately determine the indication for cystoscopy (some indications may carry a higher risk), or the use of inpatient antibiotics/duration of catheterization at the time of TURP. Also, we studied older patients undergoing THA/TKA, so our results may not be generalizable to younger patients, or other types of joint replacements. 


\section{References}

1. Shahi A, Parvizi J. Prevention of Periprosthetic Joint Infection. Arch Bone Jt Surg. 2015 Apr;3(2):72-81.

2. American Urological Association, American Academy of Orthopaedic Surgeons. Antibiotic prophylaxis for urological patients with total joint replacements. Vol. 169, Journal of Urology. 2003. pp. 1796-7.

3. Mohee AR, Gascoyne-Binzi D, West R, Bhattarai S, Eardley I, Sandoe JAT. Bacteraemia during Transurethral Resection of the Prostate: What Are the Risk Factors and Is It More Common than We Think? PLoS ONE. 2016;11(7):e0157864.

4. Almallah YZY, Rennie CDC, Stone JJ, Lancashire MJM. Urinary tract infection and patient satisfaction after flexible cystoscopy and urodynamic evaluation. Urology. 2000 Jul 1;56(1):37-9.

5. Mossanen M, Calvert JK, Holt SK, James AC, Wright JL, Harper JD, et al. Overuse of antimicrobial prophylaxis in community practice urology. J Urol. 2015 Feb;193(2):543-7.

6. Punjani N, Lanting B, McClure JA, Winick-Ng J, Welk B. The Impact of Common Urologic Complications on the Risk of a Periprosthetic Joint Infection. J Bone Joint Surg Am. 2018 Sep 5;100(17):1517-23.

7. Mrkobrada M, Ying I, Mokrycke S, Dresser G, Elsayed S, Bathini V, et al. CUA Guidelines on antibiotic prophylaxis for urologic procedures. Can Urol Assoc J. 2015 Jan;9(1-2):13-22. 
Figures and Tables

\begin{tabular}{|c|c|c|c|c|c|c|c|c|}
\hline & \multicolumn{3}{|c|}{ Cystoscopy } & \multicolumn{4}{|c|}{ Transurethral prostatectomy } & \multirow[b]{2}{*}{ SD $^{*}$} \\
\hline & Entire cohort & No & Yes & SD $^{*}$ & $\begin{array}{l}\text { Entire } \\
\text { cohort }\end{array}$ & No & Yes & \\
\hline & $\mathrm{n}=113061$ & $\mathrm{n}=84805$ & $\mathrm{n}=28256$ & & $\mathrm{n}=43461$ & $\mathrm{n}=42366$ & $n=1095$ & \\
\hline Age & $74(70-79)$ & $\begin{array}{c}74 \\
(70-79)\end{array}$ & $\begin{array}{c}75 \\
(71-79)\end{array}$ & 0.13 & $\begin{array}{c}74 \\
(70-78)\end{array}$ & $\begin{array}{c}74 \\
(70-78)\end{array}$ & $\begin{array}{c}76 \\
(72-80)\end{array}$ & 0.33 \\
\hline Female & $\begin{array}{c}69104 \\
(61.1 \%)\end{array}$ & $\begin{array}{c}65845 \\
(62.9 \%)\end{array}$ & $\begin{array}{c}3259 \\
(38.7 \%)\end{array}$ & 0.50 & 0 & 0 & 0 & 0 \\
\hline $\begin{array}{l}\text { Charlson } \\
\text { Comorbidity } \\
\text { Index }\end{array}$ & $0(0-0)$ & $0(0-0)$ & $0(0-1)$ & 0.27 & $0(0-0)$ & $0(0-0)$ & $0(0-0)$ & 0.10 \\
\hline \multicolumn{9}{|c|}{ Anesthetic for THA/TKA } \\
\hline General & $\begin{array}{c}28209 \\
(25.0 \%)\end{array}$ & $\begin{array}{c}26045 \\
(24.9 \%)\end{array}$ & $\begin{array}{c}2164 \\
(25.7 \%)\end{array}$ & 0.02 & $\begin{array}{c}10153 \\
(23.4 \%)\end{array}$ & $\begin{array}{c}9886 \\
(23.3 \%)\end{array}$ & $\begin{array}{c}267 \\
(24.4 \%)\end{array}$ & 0.02 \\
\hline Spinal & $\begin{array}{c}83820 \\
(74.1 \%)\end{array}$ & $\begin{array}{c}77633 \\
(74.2 \%)\end{array}$ & $\begin{array}{c}6187 \\
(73.4 \%)\end{array}$ & 0.02 & $\begin{array}{c}32876 \\
(75.6 \%)\end{array}$ & $\begin{array}{c}32056 \\
(75.7 \%)\end{array}$ & $\begin{array}{c}820 \\
(74.9 \%)\end{array}$ & 0.02 \\
\hline Diabetes & $\begin{array}{c}28617 \\
(25.3 \%)\end{array}$ & $\begin{array}{c}26173 \\
(25.0 \%)\end{array}$ & $\begin{array}{c}2444 \\
(29.0 \%)\end{array}$ & 0.09 & $\begin{array}{c}12505 \\
(28.8 \%)\end{array}$ & $\begin{array}{c}12197 \\
(28.8 \%)\end{array}$ & $\begin{array}{c}308 \\
(28.1 \%)\end{array}$ & 0.01 \\
\hline $\begin{array}{l}\text { Morbid } \\
\text { obesity }\end{array}$ & $7328(6.5 \%)$ & $\begin{array}{c}6801 \\
(6.5 \%)\end{array}$ & $\begin{array}{c}527 \\
(6.3 \%)\end{array}$ & 0.01 & $\begin{array}{c}2030 \\
(4.7 \%)\end{array}$ & $\begin{array}{c}1989 \\
(4.7 \%)\end{array}$ & $41(3.7 \%)$ & 0.05 \\
\hline $\begin{array}{l}\text { Peripheral } \\
\text { vascular } \\
\text { disease }\end{array}$ & $1227(1.1 \%)$ & $\begin{array}{c}1110 \\
(1.1 \%)\end{array}$ & $\begin{array}{c}117 \\
(1.4 \%)\end{array}$ & 0.03 & $\begin{array}{c}708 \\
(1.6 \%)\end{array}$ & $\begin{array}{c}689 \\
(1.6 \%)\end{array}$ & $19(1.7 \%)$ & 0.01 \\
\hline \multicolumn{9}{|c|}{ History in the past year of: } \\
\hline Urinary retenti & $\begin{array}{c}3196 \\
(2.8 \%)\end{array}$ & $\begin{array}{c}2428 \\
(2.3 \%)\end{array}$ & $\begin{array}{c}768 \\
(9.1 \%)\end{array}$ & 0.30 & $\begin{array}{c}1666 \\
(3.8 \%)\end{array}$ & $\begin{array}{c}1582 \\
(3.7 \%)\end{array}$ & $\begin{array}{c}84 \\
(7.7 \%)\end{array}$ & 0.17 \\
\hline Cystoscopy & $\begin{array}{c}4455 \\
(3.9 \%)\end{array}$ & $\begin{array}{c}2750 \\
(2.6 \%)\end{array}$ & $\begin{array}{c}1705 \\
(20.2 \%)\end{array}$ & 0.58 & $\begin{array}{c}2595 \\
(6.0 \%)\end{array}$ & $\begin{array}{c}2418 \\
(5.7 \%)\end{array}$ & $\begin{array}{c}177 \\
(16.2 \%)\end{array}$ & 0.34 \\
\hline Urinary infecti & $\begin{array}{c}13,230 \\
(11.7 \%)\end{array}$ & $\begin{array}{c}11,142 \\
(10.6 \%)\end{array}$ & $\begin{array}{c}2,088 \\
(24.8 \%)\end{array}$ & 0.38 & $\begin{array}{c}3,964 \\
(9.1 \%)\end{array}$ & $\begin{array}{c}3,746 \\
(8.8 \%)\end{array}$ & $\begin{array}{c}218 \\
(19.9 \%)\end{array}$ & 0.32 \\
\hline
\end{tabular}


Risk of infected prosthetic joint after cystoscopy or TURP

\begin{tabular}{|l|c|c|c|c|c|c|c|c|c|}
\hline $\begin{array}{l}\text { Prior antibiotic } \\
\text { exposure }\end{array}$ & $\begin{array}{c}42 \text { 265 } \\
(37.4 \%)\end{array}$ & $\begin{array}{c}38072 \\
(36.4 \%)\end{array}$ & $\begin{array}{c}4193 \\
(49.8 \%)\end{array}$ & 0.27 & $\begin{array}{c}14683 \\
(33.8 \%)\end{array}$ & $\begin{array}{c}14234 \\
(33.6 \%)\end{array}$ & $\begin{array}{c}449 \\
(41.0 \%)\end{array}$ & 0.15 \\
\hline $\begin{array}{l}\text { Prior } \\
\text { corticosteroid } \\
\text { exposure }\end{array}$ & $\begin{array}{c}19680 \\
(17.4 \%)\end{array}$ & $\begin{array}{c}18148 \\
(17.3 \%)\end{array}$ & $\begin{array}{c}1532 \\
(18.2 \%)\end{array}$ & 0.02 & $\begin{array}{c}6518 \\
(15.0 \%)\end{array}$ & $\begin{array}{c}6338 \\
(15.0 \%)\end{array}$ & $\begin{array}{c}180 \\
(16.4 \%)\end{array}$ & 0.04 \\
\hline \multicolumn{2}{|l|}{\begin{tabular}{l|l|c|c|c|c|c|} 
Number in the prior year of: \\
\hline Urology visits
\end{tabular}} & $0(0-0)$ & $0(0-0)$ & $0(0-1)$ & 0.71 & $0(0-0)$ & $0(0-0)$ & $0(0-2)$ & 0.46 \\
\hline GP visits & $7(4-10)$ & $6(4-10)$ & $7(4-11)$ & 0.15 & $6(4-10)$ & $6(4-10)$ & $7(4-10)$ & 0.13 \\
\hline Hospitalizations & $0(0-0)$ & $0(0-0)$ & $0(0-0)$ & 0.15 & $0(0-0)$ & $0(0-0)$ & $0(0-0)$ & 0.08 \\
\hline ER visits & $0(0-1)$ & $0(0-1)$ & $0(0-1)$ & 0.17 & $0(0-1)$ & $0(0-1)$ & $0(0-1)$ & 0.14 \\
\hline
\end{tabular}

All data is $n$ (proportion) or median (interquartile range). *Standardized differences (SD) are used to identify potentially clinically meaningful differences between groups; a SD $>0.10$ is considered significant, and was adjusted for in the analysis. ER: emergency room; GP: general practitioner; THA/TKA: total hip arthroplasty/total knee arthroplasty. 
Table 2. Number of patients who experienced a prosthetic joint infection based on exposure to cystoscopy or transurethral prostatectomy

\begin{tabular}{|c|c|c|c|c|}
\hline & \multicolumn{2}{|c|}{ Cystoscopy } & \multicolumn{2}{|r|}{ TP } \\
\hline & No & Yes & No & Yes \\
\hline & $(n=104635)$ & $(n=8426)$ & $(n=42366)$ & $(n=1095)$ \\
\hline $\begin{array}{l}\text { Number of persons with } \\
\text { prosthetic joint infection }\end{array}$ & 999 & 30 & 482 & $<6^{*}$ \\
\hline $\begin{array}{l}\text { Median time to first } \\
\text { cystoscopy/TP in days } \\
\text { (IQR) }\end{array}$ & & $274(107-490)$ & & 251 (84-492) \\
\hline $\begin{array}{l}\text { Unadjusted analysis } \\
\text { (HR; 95\% CI) }\end{array}$ & 1.00 (ref) & $\begin{array}{c}1.19 \\
(1.00-1.43 ; \\
\mathrm{p}=0.02)\end{array}$ & 1.00 (ref) & $\begin{array}{c}3.55 \text { (1.34-9.42; } \\
p=0.01)\end{array}$ \\
\hline $\begin{array}{l}\text { Adjusted analysis } \\
\text { (HR; 95\% CI) }\end{array}$ & 1.00 (ref) & $\begin{array}{c}1.05 \\
0.85-1.30 \\
p=0.66)^{* *}\end{array}$ & 1.00 (ref) & $\begin{array}{c}3.42(1.29-9.10 ; \\
\mathrm{p}=0.01)^{* * *}\end{array}$ \\
\hline
\end{tabular}

${ }^{*}$ Groups of people with an $\mathrm{n}<6$ are not reported in keeping with privacy regulations. ${ }^{* *}$ Adjusted for age, gender, Charlson Comorbidity Index, prior history of urinary retention/cystoscopy/urinary infection/antibiotic use and healthcare utilization. ${ }^{* * *}$ Adjusted for the same factors as above, except for gender. CI: confidence interval; HR: hazard ratio; IQR: interquartile range; TP: transurethral prostatectomy 